\title{
Linking Factors Contribute to Work Engagement Among Online Food Delivering Executives with Special Reference to Coimbatore
}

\author{
Brindha.S, R.T.Induji, C.N.Dheepenthra, S.Aravindchockalingam
}

\begin{abstract}
A positive engagement model helps to bring a positive outcome among the employees. Study shows that employees with high work engagement are energetic and dedicated to work. The concept is also related with job satisfaction. It shows that the employees with high work engagement are highly satisfied with the job. It also reveals that work engagement is positively correlated with productivity. Employees with high work engagement are highly productive in their work. Online food delivering industry is a booming industry and demands people with high productivity. The article discusses about the role of work engagement among online food delivering executives. Multiple regression analysis was performed in order to identify the variables which contribute to the work engagement among online food delivering executives. Descriptive analysis was done to know the demographic profile of the online food delivering executives
\end{abstract}

\section{OBJECTIVES}

- To know the work engagement level among the online food delivering executives

- To know the level of commitment among the online food delivering executives

\section{RESEARCH METHODOLOGY}

Descriptive research design was adopted. The sampling method adopted for the study was convenient random sampling method. Both primary and secondary data collection method was used in the study.

\section{ANALYSIS \& RESULTS}

Demographic analysis:

Educational Qualification:

\begin{tabular}{|l|r|r|r|r|}
\hline & Frequency & Percent & $\begin{array}{c}\text { Valid } \\
\text { Percent }\end{array}$ & $\begin{array}{c}\text { Cumulative } \\
\text { Percent }\end{array}$ \\
\hline $15-20$ yrs & 6 & 12.0 & 12.0 & 12.0 \\
$21-25$ yrs & 23 & 46.0 & 46.0 & 58.0 \\
$26-30$ yrs & 15 & 30.0 & 30.0 & 88.0 \\
$31-35$ yrs & 3 & 6.0 & 6.0 & 94.0 \\
Above 36 & 3 & 6.0 & 6.0 & 100.0 \\
yrs & 50 & 100.0 & 100.0 & \\
Total & & & \\
\hline
\end{tabular}

Majority of the respondents are between $21-25$ years

Revised Manuscript Received on September 14, 2019.

Dr.Brindha.S, HOD, GRD Institute of Management, Coimbatore, Tamil Nadu, India.

Mrs.R.T.Induji, Assistant Professor, Sri Krishna Arts and Science College, Coimbatore, Tamil Nadu, India.

C.N.Dheepenthra, Student, Sri Krishna Arts and Science College, Coimbatore, Tamil Nadu, India.

S.Aravindchockalingam,
Educational qualification

\begin{tabular}{|l|r|r|r|r|}
\hline & $\begin{array}{c}\text { Frequenc } \\
\mathrm{y}\end{array}$ & Percent & $\begin{array}{r}\text { Valid } \\
\text { Percent }\end{array}$ & $\begin{array}{c}\text { Cumulative } \\
\text { Percent }\end{array}$ \\
\hline 10th std & 6 & 12.0 & 12.0 & 12.0 \\
12 th std & 3 & 6.0 & 6.0 & 18.0 \\
UG & 35 & 70.0 & 70.0 & 88.0 \\
PG & 3 & 6.0 & 6.0 & 94.0 \\
below 10th std & 3 & 6.0 & 6.0 & 100.0 \\
Total & 50 & 100.0 & 100.0 & \\
\hline
\end{tabular}

Majority of the respondents are undergraduates.

Income

\begin{tabular}{|l|r|r|r|r|}
\hline & $\begin{array}{r}\text { Frequenc } \\
\mathrm{y}\end{array}$ & Percent & $\begin{array}{r}\text { Valid } \\
\text { Percent }\end{array}$ & $\begin{array}{c}\text { Cumulative } \\
\text { Percent }\end{array}$ \\
\hline $\begin{array}{l}6000- \\
15000 \\
16000-\end{array}$ & 24 & 48.0 & 48.0 & 48.0 \\
25000 & 15 & 30.0 & 30.0 & 78.0 \\
$26000-$ & 3 & 6.0 & 6.0 & 84.0 \\
35000 & 8 & 16.0 & 16.0 & 100.0 \\
Above & 50 & 100.0 & 100.0 & \\
35000 & & & & \\
Total & & & & \\
\hline
\end{tabular}

\section{Income}

Majority of the respondent's income is between Rs.6000 - Rs. 15000

Gender

\begin{tabular}{|c|r|r|r|r|}
\hline & $\begin{array}{c}\text { Frequenc } \\
\mathrm{y}\end{array}$ & Percent & $\begin{array}{c}\text { Valid } \\
\text { Percent }\end{array}$ & $\begin{array}{c}\text { Cumulative } \\
\text { Percent }\end{array}$ \\
\hline male & 41 & 82.0 & 82.0 & 82.0 \\
female & 9 & 18.0 & 18.0 & 100.0 \\
Total & 50 & 100.0 & 100.0 & \\
\hline
\end{tabular}

Gender

Majority of the respondents are male.

Published By: 


\section{Multiple regression analysis:}

Multiple regression analysis was performed to check the work engagement level. Work engagement was the outcome variable and it was tested with the predictor variables like work was meaningful, time, enthusiasm, forgetting everything, inspiring, feeling happy, proud, involvement.

\section{Descriptive Statistics}

\begin{tabular}{|l|r|r|r|}
\hline & Mean & Std. Deviation & $\mathrm{N}$ \\
\hline work engagement & 3.5000 & 1.70533 & 50 \\
meaningful and purpose & 3.7600 & 1.58514 & 50 \\
Time flies at work & 4.2200 & 1.61990 & 50 \\
strong and vigorous & 4.1200 & 1.43769 & 50 \\
Enthusiasm & 4.1800 & 1.40973 & 50 \\
forgetting everything & 4.7200 & 1.08872 & 50 \\
Inspiring & 4.6200 & 1.19335 & 50 \\
feeling to go to work & 4.1000 & 1.94044 & 50 \\
Feeling happy & 4.2000 & 1.52530 & 50 \\
Proud & 4.6000 & 1.39971 & 50 \\
Involvement & 4.6000 & 1.39971 & 50 \\
Willing to work & 4.8200 & .87342 & 50 \\
\hline
\end{tabular}

Model Summaryb

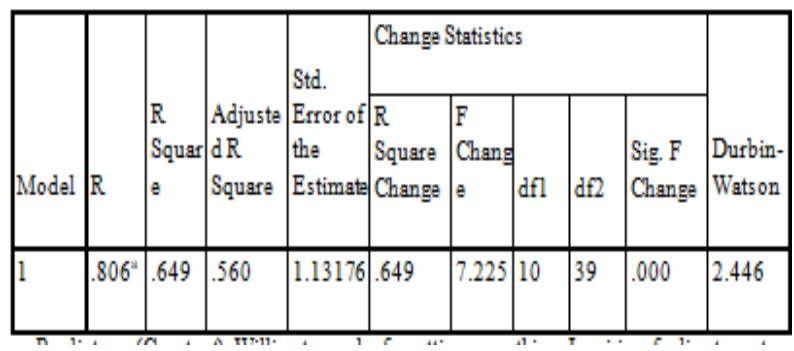

a. Predictors: (Constant), Willing to work, forgetting everything,

Inspiring, feeling to go to work, strong and vigorous, Time flies at work,

Feeling happy, Enthusiasm, meaningful and purpose, Involvement From the study it is concluded that the predictor variables

b. Dependent Variable:

work engagement

The $\mathrm{R}$ value is the simple correlation value. $\mathrm{R}$ square is the extent of variation brings about by the predictor variable on the outcome variable. Adjusted R square gives some idea of how well the model generalizes

ANOVA

\begin{tabular}{|c|c|c|c|c|c|c|}
\hline \multicolumn{2}{|c|}{ Model } & $\begin{array}{l}\text { Sum of } \\
\text { Squares }\end{array}$ & df & $\begin{array}{l}\text { Mean } \\
\text { Square }\end{array}$ & $\mathrm{F}$ & Sig. \\
\hline 1 & Regression & 92.545 & 10 & 9.255 & 7.225 & $.000^{\circ}$ \\
\hline & Residual & 49.955 & 39 & 1.281 & & \\
\hline & Total & 142.500 & 49 & & & \\
\hline
\end{tabular}

a. Predictors: (Constant), Willing to work , forgetting everything, Inspiring, feeling to go to work, strong and vigorous, Time flies at work, Feeling happy, Enthusiasm, meaningful and purpose, Involvement

b. Dependent Variable: work engagement
The result shows there is significant difference between the predictor variable and the outcome variable among the online food delivering executives.

The result shows that the predictor variables willing to work at any time, forgetting everything at work, Inspiring, feeling to go to work everyday, feeling strong and vigorous, time flies when at work, feeling happy at work, enthusiasm, work was meaningful and involvement significantly contribute to engaging the employees at work.

\begin{tabular}{|c|c|c|c|c|c|c|c|}
\hline \multirow[b]{2}{*}{ Model } & \multicolumn{2}{|c|}{$\begin{array}{l}\text { Unstandardized } \\
\text { Coefficients }\end{array}$} & \multirow{2}{*}{\begin{tabular}{|l}
$\begin{array}{l}\text { Standar } \\
\text { dized } \\
\text { Coeffici } \\
\text { ents }\end{array}$ \\
Beta
\end{tabular}} & \multirow[b]{2}{*}{$\mathrm{t}$} & \multirow[b]{2}{*}{ Sig. } & \multicolumn{2}{|c|}{$\begin{array}{l}\text { Collinearity } \\
\text { Statistics }\end{array}$} \\
\hline & B & $\begin{array}{l}\text { Std. } \\
\text { Error }\end{array}$ & & & & $\begin{array}{l}\text { Toler } \\
\text { ance }\end{array}$ & VIF \\
\hline $1 \quad$ (Constant) & 2.960 & 1.495 & & 1.980 & .055 & & \\
\hline $\begin{array}{l}\text { meaningful } \\
\text { and purpose }\end{array}$ & 1.024 & .179 & .952 & 5.709 & .000 & .323 & 3.095 \\
\hline $\begin{array}{l}\text { Time flies at } \\
\text { work }\end{array}$ & .110 & .143 & .105 & .770 & .446 & .486 & 2.058 \\
\hline $\begin{array}{l}\text { strong and } \\
\text { vigorous }\end{array}$ & -.090 & .154 & -.076 & -.589 & .559 & .536 & 1.866 \\
\hline Enthusiasm & -.005 & .220 & -.004 & -.024 & .981 & .272 & 3.670 \\
\hline $\begin{array}{l}\text { forgetting } \\
\text { everything }\end{array}$ & -.408 & .244 & -.260 & 1.668 & .103 & .369 & 2.707 \\
\hline Inspiring & -.019 & .141 & -.013 & -.136 & .892 & .926 & 1.080 \\
\hline $\begin{array}{l}\text { feeling to go } \\
\text { to work }\end{array}$ & -.097 & .125 & -.110 & -.774 & .444 & .442 & 2.261 \\
\hline Feeling happy & -.265 & .142 & -.237 & 1.874 & .068 & .561 & 1.784 \\
\hline Involvement & .105 & .241 & .086 & .438 & .664 & .230 & 4.343 \\
\hline $\begin{array}{l}\text { Willing to } \\
\text { work }\end{array}$ & -.071 & .304 & -.036 & -.234 & .816 & .371 & 2.697 \\
\hline
\end{tabular}

a. Dependent Variable: work engagement significantly contribute to the outcome variable. Most importantly the study shows that the online food delivering executives understand the purpose and feel the meaningfulness of the work. 